\title{
Pars Plana Lensectomy and Iris-Claw Artisan Intraocular Lens Implantation in Patients with Marfan Syndrome
}

Saeed Karimi

Shahid Beheshti University of Medical Sciences

Zahra Karjou ( $\sim$ zahrakarjuo@yahoo.com)

Shahid Beheshti University of Medical Sciences

Mohadeseh Yaghoobi

Shahid Beheshti University of Medical Sciences

Homayoun Nikkhah

Shahid Beheshti University of Medical Sciences

Sare Safi

Shahid Beheshti University of Medical Sciences

\section{Research Article}

Keywords: Marfan syndrome, Crystalline lens subluxation, Pars plana lensectomy, Iris-claw artisan intraocular lens

Posted Date: June 8th, 2021

DOI: https://doi.org/10.21203/rs.3.rs-519533/v1

License: (c) (i) This work is licensed under a Creative Commons Attribution 4.0 International License. Read Full License 


\section{Abstract}

Purpose: To evaluate the visual and anatomical outcomes of pars plana lensectomy and iris-claw artisan intra-ocular lens (IOL) implantation in the patients with subluxated crystalline lens secondary to Marfan syndrome.

Methods: In this retrospective case series, we evaluate the records of all patients with Marfan syndrome and moderate to severe crystalline lens subluxation who underwent pars plana lensectomy/anterior vitrectomy and implantation of iris-claw artisan IOL at referral hospital from September 2015 to October 2019.

Results: Twenty-one eyes of fifteen patients (10 males and 5 females) with the mean age of $24.47 \pm$ 19.14 years were included. Mean best-corrected visual acuity was improved from $1.17 \pm 0.55 \log M A R$ to $0.64 \pm 0.71 \log M A R$ at the final follow-up visit $(P<0.001)$. The mean intraocular pressure did not change significantly $(P=0.971)$. The final refraction showed a mean sphere of $0.54 \pm 2.46 \mathrm{D}$ and a mean cylinder $-0.81 \pm 1.03$ at the mean axis of $57.92 \pm 58.33$ degrees. One eye developed rhegmatogenous retinal detachment two months after surgery.

Conclusion: Pars plana lensectomy and iris-claw artisan IOL implantation seem to be a useful, impressive and safe procedure with a low rate of complications in Marfan patients with moderate to severe crystalline lens subluxation. Visual acuity was significantly improved with the acceptable anatomical and refractive outcomes.

\section{Introduction}

Marfan syndrome is a genetic disorder that affects the connective tissue and is caused by a mutation in the FBN1 gene on chromosome 15. The FBN1 gene encodes a fibrillin-1 protein which is a principal component of zonules, and its defect result in ectopia lentis (50\%-80\% cases) [1-2]. Marfan syndrome is a systemic disease that classically affects the cardiovascular, musculoskeletal, and ocular systems. For ocular diagnosis of Marfan syndrome, one major or two minor criteria should exist[3]. Lens subluxation is a major ocular criterion; flat cornea (as measured by keratometry)[4], increased axial length of globe, and hypoplastic ciliary muscle or iris are minor criteria. Other diagnostic ocular findings include retinal detachment, high myopia, cataract, glaucoma, strabismus, and amblyopia[5-9], Ectopia lentis may result in refractive errors such as astigmatism, myopia, or aphakic hyperopia. Patients may experience diplopia if the equator of the lens crosses the visual axis[2]. Initial management is conservative with refractive correction and amblyopia therapy. However, surgery is recommended when it is difficult to obtain functional visual acuity with a nonsurgical approach, or when severe lens subluxation or posterior dislocation causes unstable refractive status, or if lens dislocation to the anterior chamber causes secondary IOP rise or endothelial compromise[2, 10-11]. The best surgical approach is still controversial. The limbal approach or pars plana approach may be chosen. The surgeon's comfort level can be an important factor in any method[12]. In the presence of large lens subluxation, it is very difficult to place a 
posterior chamber intraocular lens (IOL) even with the support of a capsule tension ring (CTR) or Cionni ring, although there is the possibility of ring and IOL dislocation[12-13]. For aphakia correction, various IOLs can be used, such as angle-supported anterior chamber IOLs, scleral fixation posterior chamber intraocular lenses (SF-PCIOL), and, more recently, retro-pupillary iris-claw IOLs[14]. These cases have a tendency to avoid angle-supported anterior chamber, because of the potential risk of secondary glaucoma and long-term endothelial damage[13, 15]. Iris-claw Artisan implantation is the optimal choice for aphakia correction with several advantages such as good visual outcome, fewer complications, and easy placement $[14,16-17]$.

We evaluated the visual and anatomical outcomes of pars plana lensectomy with implantation of artisan $\mathrm{IOL}$ in eyes with subluxated lenses in Marfan syndrome.

\section{Materials And Methods}

In this retrospective non-comparative case series, we evaluate the records of all patients with Marfan syndrome and subluxated crystalline lens who needed pars plana lensectomy with the implantation of iris-claw artisan IOL at a referral hospital from September 2015 to October 2019. The study was performed based on the Declaration of Helsinki and was approved by the Institutional Ethics Committee. We included only patients with at least 3 months of follow-up. The patients with a history of previous intraocular surgery, missing complete pre-and post-operative records, and lens dislocation into the vitreous cavity was excluded from the study. Preoperative data included the demographic details, main preexisting ocular pathology, Snellen visual acuity, refraction, and intraocular pressure (IOP). Surgical technique, intraoperative problems, and postoperative information, including final best-corrected visual acuity (BCVA), IOP, refraction, and incidence of complications, were obtained. IOL power was measured using laser interferometry (IOL Master500; Zeiss, Jena, Germany). Based on the axial length, IOL power calculation was done using the SRK/T, Hoffer, or Holladay formula. In all cases, our target refraction was emmetropia. All procedures were performed by one surgeon under general anesthesia. Lensectomy was performed using the pars plana approach. In this approach, $3 \mathrm{~mm}$ from the limbus, two sclerotomies were made. A vitreous cutter probe was used to perform a complete lensectomy to ensure that all capsular residues were removed. Subsequently, anterior vitrectomy and peripheral retinal examination were done to discover any unintentional retinal breaks. The sclerotomies were secured with $8-0$ vicryl suture. Finally, pupil constriction was achieved by carbachol $0.01 \%$, and peripheral iridectomy was performed. A limbal incision was made at the 12-o'clock position and two ports at the 10- and 2-o'clock positions. The aphakic artisan IOL (Ophtec, Groningen, Netherlands) was inserted into the anterior chamber and fixed to the iris with an enclavation needle (Ophtec). At the end of the surgery, anterior chamber washing was done, and the wound was sutured with $10-0$ nylon. Postoperatively topical antibiotics and steroid drops were prescribed and tapered for 4 weeks. Selective corneal sutures removal according to corneal astigmatism were performed 6 to 8 postoperative weeks. BCVA, anterior, and posterior segment examination and intraocular pressure, were documented on follow-up visits.

\section{Statistical Analysis}


A commercially available statistical software package (SPSS version 23; IBM Corp., New York, NY, USA) was used to perform univariate analyses using parametric and nonparametric methods. For qualitative variables, frequencies and proportions (as percentages) were calculated. For normally distributed quantitative variables, the mean and standard deviation were calculated. For quantitative variables which were not normally distributed, the median and $25 \%$ quartile was calculated. Statistical significance was defined as $\mathrm{P}<0.05$.

\section{Results}

Twenty-one eyes of fifteen patients ( 10 males and 5 females) with Marfan syndrome and severe crystalline lens subluxation were included. Thirteen eyes were from male patients, and 8 eyes were from female patients. The mean age of patients was $24.47 \pm 19.14$ years. Mean BCVA significantly improved from $1.17 \pm 0.55 \log M A R$ preoperatively to $0.64 \pm 0.71 \log M A R$ at final follow-up visit $(P<0.001)$. The mean follow-up duration was $14.7 \pm 5.2$ months.

Mean intraocular pressure (IOP) before the surgery was $15.15 \pm 7.4$. Mean IOP at the last follow-up after the surgery was $13.5 \pm 2.7 \mathrm{mmHg}$, respectively $(P=0.971)$.

Pre-operative refractive error was unstable and invaluable. The mean final postoperative spherical equivalent was $+0.14 \pm 2.37$ diopters $(D)$. the constituent components of refraction were evaluated using power vector analysis that showed a mean sphere of $0.54 \pm 2.46 \mathrm{D}$ and a mean cylinder $-0.81 \pm 1.03$ at a mean axis of $57.92 \pm 58.33$ degrees. There was no significant early postoperative complication. Only one case developed rhegmatogenous retinal detachment (RRD) two postoperative months and was approached by standard three-port pars plana deep vitrectomy and silicone oil tamponade. Visual acuity improvement occurred in all eyes except three which seems to be caused by preoperative amblyopia. We did not observe clinically significant IOP elevation or other complications such as pupillary block, uveitis, IOL dislocation, endophthalmitis, macular edema, or endothelial decompensation in follow-up visits.

\section{Discussion}

The present study showed the feasibility, effectiveness, and safety of pars plana lensectomy and anterior iris-claw IOL implantation in patients with moderate to severe crystalline lens subluxation in Marfan syndrome. Our results showed significant improvement in visual acuity and acceptable refractive outcomes postoperatively. This improvement was achieved in the first postoperative weeks and then remained almost stable throughout the follow-up visits. Comparing pre and postoperative values, IOP did not change significantly. The development of amblyopia in children with large lens subluxations can be a factor limiting postoperative visual gain when the surgery was performed after childhood. Three of our patients did not show significant improvement in visual acuity because of amblyopia.

The management of ectopia lentis differs based on the preoperative and intraoperative findings. The lens subluxation grade plays an important role. In cases of mild lens subluxation, phacoemulsification and 
CTR supported, in the bag IOL implantation can be performed. In all of our cases, the grade of the lens subluxation was moderate or severe. All patients were managed with pars plana lensectomy/anterior vitrectomy followed by anterior iris-claw artisan IOL implantation. This approach has advantages over phacoemulsification, particularly in the complex eyes with specific challenges including difficult capsulorhexis, phacoemulsification in the content of unstable and floating lens and its capsule, and also insertion of CTR or fixation of Cionni ring. Furthermore, pars plana lensectomy and anterior vitrectomy can avoid intraoperative complications of phacoemulsification technique such as lens capsular bag compromise or vitreous traction and postoperative in-bag IOL dislocation, especially in severe cases [1819].

Artisan implantation is an effective method to manage the aphakia in the absence of capsular support. Although anterior chamber Artisan IOL implants were associated with some complications such as iritis, IOL disenclavation, and endothelial cell compromise; numerous studies indicated that despite some endothelial cell loss in the long term, IOL and corneal endothelium keep a safe distance from each other (ranging from 2.99 to $3.01 \pm 0.56 \mathrm{~mm}$ ) [20-24]. Several studies have shown that artisan IOL had several advantages and fewer complications when compared with the transscleral sutured PCIOLs and anglesupported ACIOLs [11]. Insertion and implantation of artisan IOL are easy and visual outcomes are good. Being fixated to the midperipheral iris in a location that is away from the AC angle, artisan IOL is less likely to damage the angle structures [25-27]. The scleral-fixated IOL implantation technique may increase the occurrence of postoperative retinal detachment in patients with Marfan syndrome because these patients commonly have some peripheral retinal changes predisposing them to a rhegmatogenous retinal detachment [28]. Cleary et al. believe that the main advantage of iris-claw ACIOL over scleralfixated IOL is that manipulation of the vitreous not occurred, and the posterior segment remains intact [29]. Suture erosion and IOL subluxation or dislocation may occur in IOL suturing to sclera or iris and sutured Cionni. Such a way that IOL dislocation has been reported in up to $24 \%$ of pediatric cases [30]. Other complications include intraoperative hemorrhages, cystoid macular edema, IOL tilting, decentration or iris capture, and increased retinal detachment incidence [31-33]. Posteriorly enclavated iris-claw IOL may potentially lower endothelial cell loss, but some recent studies showed that anterior and posterior iris-claw IOL proved almost identical effectiveness and safety for aphakic correction. Surgical technique choice depends on the surgeon's trend [34-35].

In our study, only one eye developed rhegmatogenous retinal detachment two months post-operatively. Even without surgical intervention, in eyes with Marfan syndrome, the retinal detachment rate is higher than the normal population, perhaps because of high axial myopia and lens subluxation [1, 5, 36-37]. Zonular deficiency and subsequent lens subluxation in Marfan syndrome are assumed to produce a persistent pull on the Ora Serrata. Increased axial length, liquefied vitreous gel, and traction on vitreoretinal adhesion are other predisposing factors leading to retinal thinning and eventually retinal detachment [36-37]. Chandra et al. showed that twenty-one percent of Marfan patients with retinal detachment had previous subluxated lens surgery history. The authors concluded that lens subluxation surgery could be a risk factor for retinal detachment occurrence [38]. A more severe grade of lens subluxation and high axial myopia was seen in the patients who developed retinal detachment. 
Limitations of our study are retrospective design and relatively small sample size. Moreover, the endothelial cell damage was not evaluated which is a concern in artisan implantation. Since Marfan syndrome is a rare condition, multicenter studies with larger sample sizes and longer follow-up visits are recommended.

To conclude, our study showed that pars plana lensectomy and iris-claw artisan IOL implantation seems to be a safe and effective procedure with a low rate of complication in Marfan patients with moderate to severe crystalline lens subluxation. Visual acuity significantly improved, and the anatomical and refractive outcomes were acceptable.

\section{Declarations}

\section{Funding:}

None.

\section{Conflict of Interest:}

Saeed Karimi declares that he has no conflict of interest. Zahra Karjou declares that she has no conflict of interest. Mohadeseh Yaghoobi declares that she has no conflict of interest. Homayoun Nikkhah declares that he has no conflict of interest. Sare Safi declares that she has no conflict of interest.

\section{Availability of data and material:}

Data is available.

\section{Code availability:}

SPSS software (IBM Corp. SPSS version 23; IBM Corp., New York, NY, USA)

\section{Compliance with Ethical Standards:}

All procedures performed in studies involving human participants were in accordance with the ethical standards of the Institutional Research Committee and with the 1964 Helsinki declaration and its later amendments or comparable ethical standards. The Institutional Ethics Committee, Tehran, Iran, approved this study.

\section{Consent to participate:}


Informed consent was obtained from all individual participants included in the study.

\section{Consent to publish:}

Patients signed informed consent regarding publishing their data.

\section{References}

1. Nemet AY, Assia El, Apple DJ et al (2006) Current concepts of ocular manifestations in Marfan syndrome. Surv Ophthalmol 51: 561-575.

2. Esfandiari H, Ansari S, Mohammad-Rabei $\mathrm{H}$ et al (2019) Management strategies of ocular abnormalities in patients with Marfan syndrome: current perspective. J Ophthalmic Vis Res 2019; 14: 71.

3. De Paepe A, Devereux RB, Dietz HC, et al (1996) Revised diagnostic criteria for the Marfan syndrome. Am J Med Genet A 62: 417-26.

4. Heur M, Costin B, Crowe $S$ et al (2008) The value of keratometry and central corneal thickness measurements in the clinical diagnosis of Marfan syndrome. Am J Ophthalmol 145:997-1001.

5. Loewenstein A, Barequet IS, De Juan Jr E et al (2000) Retinal detachment in Marfan syndrome. Retina 20: 358-363.

6. Vanem TT. Marfan syndrome in adults: re-investigations in a Norwegian cohort after 10 years. Available from: https://www.duo.uio.no/handle/10852/84057

7. Scheibenberger D, Frings A, Steinberg J et al (2018) Ocular manifestation in Marfan syndrome: corneal biomechanical properties relate to increased systemic score points. Graefes Arch Clin Exp Ophthalmol 256:1159-1163.

8. Nahum Y, Spierer A (2008) Ocular features of Marfan syndrome: diagnosis and management. Isr Med Assoc J 10:179-181.

9. Gehle P, Goergen B, Pilger D et al (2017) Biometric and structural ocular manifestations of Marfan syndrome. PloS one 12: e0183370.

10. Kazemi S, Wirostko W, Sinha S et al (2000) Combined pars plana lensectomy-vitrectomy with openloop flexible anterior chamber intraocular lens (AC IOL) implantation for subluxated lenses. Trans Am Ophthalmol Soc 98:247.

11. Utz VM, Coussa RG, Traboulsi El (2014) Surgical management of lens subluxation in Marfan syndrome. J AAPOS 18: 140-146.

12. Simon MA, Origlieri CA, Dinallo AM et al (2015) New management strategies for ectopia lentis. $J$ AAPOS 52: 269-281.

13. Dick HB and Augustin AJ (2001) Lens implant selection with absence of capsular support. Curr Opin Ophthalmol 12: 47-57. 
14. Acar N, Kapran Z, Altan T et al (2010) Secondary iris claw intraocular lens implantation for the correction of aphakia after pars plana vitrectomy. Retina 30: 131-139.

15. Hara T, Hara T (2004) Ten-year results of anterior chamber fixation of the posterior chamber intraocular lens. Arch Ophthalmol 122:1112-1116.

16. Hara S, Borkenstein AF, Ehmer A et al (2011) Retropupillary fixation of iris-claw intraocular lens versus transscleral suturing fixation for aphakic eyes without capsular support. $J$ Refract Surg 27: 729-735.

17. Agarwal A, Kumar DA, Jacob S et al (2008) Fibrin glue-assisted sutureless posterior chamber intraocular lens implantation in eyes with deficient posterior capsules. J Cataract Refract Surg 34: $1433-1438$.

18. Hoffman RS, Snyder ME, Devgan U et al; ASCRS Cataract Clinical Committee; Challenging/Complicated Cataract Surgery Subcommittee (2013) Management of the subluxated crystalline lens. J Cataract Refract Surg 39:1904-1915.

19. Fan F, Luo Y, Liu X et al (2014) Risk factors for postoperative complications in lensectomyvitrectomy with or without intraocular lens placement in ectopia lentis associated with Marfan syndrome. Br J Ophthalmol 98: 1338-1342.

20. Català-Mora J, Cuadras D, Díaz-Cascajosa J et al (2017) Anterior iris-claw intraocular lens implantation for the management of nontraumatic ectopia lentis: long-term outcomes in a paediatric cohort. Acta ophthalmologica 95: 170-174.

21. Baikoff G (2006) Anterior segment OCT and phakic intraocular lenses: A perspective. J Cataract Refract Surg 32: 1827-1835.

22. Güell JL, Morral M, Gris $O$ et al (2007) Evaluation of Verisyse and Artiflex phakic intraocular lenses during accommodation using Visante optical coherence tomography. J Cataract Refract Surg 33: 1398-1404.

23. Moshirfar M, Holz HA, Davis DK (2007) Two-year follow-up of the Artisan/Verisyse iris-supported phakic intraocular lens for the correction of high myopia. J Cataract Refract Surg 33: 1392-1397.

24. Ferreira TB, Portelinha J (2014) Endothelial distance after phakic iris-fixated intraocular lens implantation: a new safety reference. Clin Ophthalmo/ 8:255-261.

25. Teichman $\mathrm{J}$ and Ahmed I (2007) Improved technique with the Artisan aphakic IOL. Review of Ophthalmology 14: 59.

26. Said O, Saad S, El-Hafez M et al (2019) Two Years Follow up after Iris Claw Intraocular Lens Implantation for Correction of Aphakia. J Clin Exp Ophthalmol 10: 2.

27. Zafar SN, Siddiqui SN, Khan A (2013) Effects of Artisan aphakic intraocular lens on central corneal thickness and intra ocular pressure in pediatric eyes with crystalline subluxated lenses. Oman $\mathrm{J}$ Ophthalmol 6: 44.

28. Anteby I, Isaac M, BenEzra D (2003) Hereditary subluxated lenses: visual performances and longterm follow-up after surgery. Ophthalmology 110: 1344-1348. 
29. Cleary C, Lanigan B, O'Keeffe M (2012) Artisan iris-claw lenses for the correction of aphakia in children following lensectomy for ectopia lentis. Br J Ophthalmo/ 96:419-421.

30. Buckley EG (2009) Pediatric sutured intraocular lenses: trouble waiting to happen. Am J Ophthalmol 147: 3-4.

31. Konradsen T, Kugelberg M, Zetterström C (2007) Visual outcomes and complications in surgery for ectopia lentis in children. J Cataract Refract Surg 33: 819-824.

32. Bading G, Hillenkamp J, Sachs HG et al (2007) Long-term safety and functional outcome of combined pars plana vitrectomy and scleral-fixated sutured posterior chamber lens implantation. $\mathrm{Am}$ J Ophthalmol 144: 371-377. e1.

33. Kim EJ, Berg JP, Weikert MP, et al (2014) Scleral-fixated capsular tension rings and segments for ectopia lentis in children. Am J Ophthalmol 158: 899-904. e1.

34. Toro MD, Longo A, Avitabile T et al (2019) Five-year follow-up of secondary iris-claw intraocular lens implantation for the treatment of aphakia: Anterior chamber versus retropupillary implantation. PLOS One 14:e0214140.

35. Mora P, Calzetti G, Favilla S et al (2018) Comparative Analysis of the Safety and Functional Outcomes of Anterior versus Retropupillary Iris-Claw IOL Fixation. J Ophthalmol 2018:8463569.

36. Sharma T, Gopal L, Shanmugam MP et al (2002) Retinal detachment in Marfan syndrome: clinical characteristics and surgical outcome. Retina 22:423-428.

37. Lee S-Y and Ang C-L (2003) Results of retinal detachment surgery in Marfan syndrome in Asians. Retina 23: 24-29.

38. Chandra A, Ekwalla V, Child A et al (2014) Prevalence of ectopia lentis and retinal detachment in Marfan syndrome. Acta ophthalmologica 92: e82-e3. 